\title{
Formation of self-organized platinum nanoparticles and their microphotoluminescence enhancement in the visible light region
}

\author{
Chieh-Yu Kang, Cha-Hsin Chao, and Shu-Chia Shiu \\ Graduate Institute of Electro-Optical Engineering, National Taiwan University, Taipei, Taiwan 10617, \\ Republic of China \\ Li-Jen Chou and Mu-Tung Chang \\ Department of Materials Science and Engineering, National Tsing Hua University, Hsinchu 30013, \\ Republic of China \\ Gong-Ru Lin and Ching-Fuh Lin ${ }^{\text {a) }}$ \\ Graduate Institute of Electro-Optical Engineering, Graduate Institute of Electronics Engineering, \\ and Department of Electrical Engineering, National Taiwan University, Taipei, Taiwan 10617, \\ Republic of China
}

(Received 24 February 2007; accepted 6 August 2007; published online 3 October 2007)

Formation of Pt nanoparticles or nanoisland films as a function of annealing temperature, initial thickness, underlying substrates, and annealing process is investigated. Using microphotoluminescence (PL) measurement, we find great enhancement of self-emission in visible spectrum from Pt nanoparticles. The integral intensity of the micro-PL of the $49.38 \mathrm{~nm}$ Pt nanoparticles is 38 times of that of the Pt thin film. In addition, the peak wavelength varies from 554 to $615 \mathrm{~nm}$ as the surface morphology of Pt changes due to different annealing parameters. Spectral analyses suggest that this enhancement of micro-PL from $\mathrm{Pt}$ is due to the local field enhancement mechanism analogous to that of PL from noble metals. (c) 2007 American Institute of Physics.

[DOI: $10.1063 / 1.2785942]$

\section{INTRODUCTION}

The unique optical properties of roughened metal surfaces or metal nanoparticles, especially of noble metals such as gold, silver, and copper, have been intensively studied over the past few decades. For instance, gold exhibits particular absorption peaks at visible wavelengths due to surface plasmon resonance (SPR) as its size is reduced to nanometer dimensions. ${ }^{1}$ The Raman scattering of rhodamine $6 \mathrm{G}$ or other organic molecules adsorbed on aggregated Ag colloids can also be greatly enhanced. ${ }^{2}$ This is the well-known phenomenon called surface enhanced Raman scattering (SERS).

Enhanced photoluminescence from noble metal nanoparticles has also been observed and has attracted much attention. ${ }^{3}$ Theoretical work has previously demonstrated that the photoluminescence (PL) of noble metals is the direct recombination of the conduction-band electrons near the Fermi level with the holes in the $d$ band. ${ }^{4}$ However, the PL intensity of noble metals is very weak. The PL efficiency of smooth gold explored by Mooradian's original experiment was on the order of $10^{-10}$. One possible reason for this low PL efficiency is that the nonradiative energy relaxation processes of photoexcited carriers in metals, such as Coulomb carrier-carrier scattering, are much faster than radiative electron-hole recombination, thus quenching the PL. ${ }^{5}$ However, when noble metals are reduced to sizes in the range of nanometers, PL efficiency can be enhanced by about

\footnotetext{
${ }^{\text {a) }}$ Author to whom correspondence should be addressed. Telephone: 886-23366 3540. FAX: 886-2-2364 2603. Electronic mail cflin@cc.ee.ntu.edu.tw
}

$10^{4}-10^{6}$ times. $^{6}$ PL enhancement based on the phenomenological model by Boyd et al. ${ }^{7}$ has been considered due to local field enhancement. Nonetheless, some scientists have developed different ideas in recent years. Dulkeith et al. explained a process in which the excited $d$-band holes recombined with the $s p$ electrons emitting plasmons. These plasmons subsequently radiated, giving rise to PL. ${ }^{8}$

For transition metals, previous investigation has shown that their optical properties when they are several nanometers in size cannot be enhanced as significantly as those observed in $\mathrm{Au}, \mathrm{Ag}$, and $\mathrm{Cu}^{9}{ }^{9}$ However, many applications and studies have been focused on them, particularly on $\mathrm{Pt}$, which has been often used as electrode material. For example, roughened platinum electrodes can enhance the Raman scattering of pyridine ${ }^{10}$ or other organic molecules; platinum island films can also enhance light absorption of some materials, such as infrared absorption of $p$-NTP. ${ }^{11}$ A recent report demonstrated that $\mathrm{Pt}$ nanoparticles sputtered on $\mathrm{ZnO}$ can greatly enhance the bandgap emission of $\mathrm{ZnO}$ nanorods. ${ }^{12}$ In this paper, we present an annealing method for fabricating Pt nanoisland films and nanoparticles of different sizes. The formation of nanoscale Pt particles as a function of temperature, film thickness, underlying substrates, and annealing process is reported. We have observed that the peak wavelength of surface enhanced micro-PL of Pt was shifted by the changes in the particles' shapes. X-ray photoelectron spectroscopy (XPS) and X-ray diffraction (XRD) were utilized to examine the composition of our samples. In order to observe the surface plasmon effect on our samples, the extinction spectra of Pt nanoisland films were measured. 


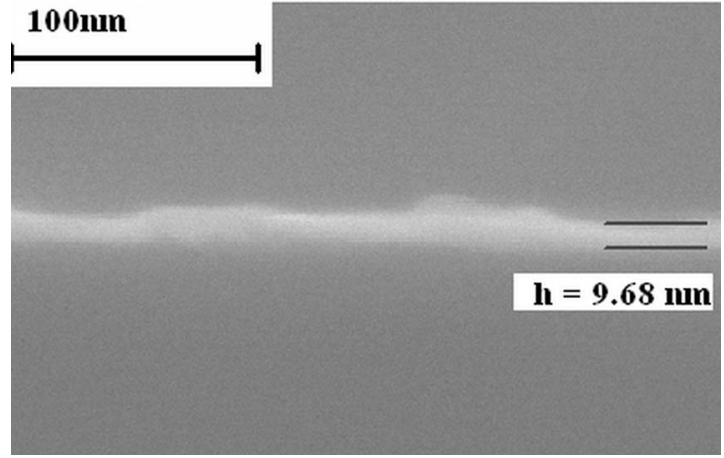

FIG. 1. SEM picture of unannealed Pt film.

\section{EXPERIMENT}

We produced our samples using platinum nanoparticles or nanoisland films on $\mathrm{Si}$ or quartz substrates. Si substrates were initially cleaned by acetone, methanol, and de-ionized water sequentially. Then, spin-on glass (SOG) $31 \mathrm{~F}$ was spun on silicon substrates. The platinum film was deposited on SOG film by electron-gun evaporation at $1.8 \times 10^{-6}$ torr. The deposition rate was estimated to be $0.01 \mathrm{~nm} / \mathrm{s}$. The scanning electron microscopy (SEM) photo of the unannealed Pt film is shown in Fig. 1. Its thickness is about $10 \mathrm{~nm}$. The samples with the Pt thin film were then annealed at different temperatures. Different annealing apparatuses had also been tested. One used the rapid thermal annealing (RTA) with $\mathrm{N}_{2}$ purging (RTA process) and the other used a heating stage in a chamber under a pressure of $10^{-3}$ torr (vacuum process). The purpose of the SOG film spun on the silicon substrates was to prevent the formation of PtSi alloy or other platinum silicides when the Pt thin film was annealed. ${ }^{13}$ The samples on the quartz substrates were prepared by the same procedure as those on the Si substrates. After annealing for $5 \mathrm{~min}, \mathrm{Pt}$ islands in the nanometer range were formed. The morphology and size distribution of the Pt nanoisland films were investigated using SEM. Micro-PL spectra of Pt nanoisland films were measured using excitation from a $514.5 \mathrm{~nm} \mathrm{Ar}^{+}$laser focused through a microscope. The radiative emission was dispersed by a monochromator and detected by a cooled photomultiplier tube (PMT). The XRD studies were measured with a $\mathrm{Cu} \mathrm{K} \alpha$ line and the XPS studies were carried out with an $\mathrm{Mg} \mathrm{K} \alpha \mathrm{X}$-ray source $(1253.6 \mathrm{eV})$. The extinction spectra of the platinum nanoisland films were also measured for the samples fabricated on the quartz substrates by a spectrophotometer with a resolution of $0.5 \mathrm{~nm}$.

\section{RESULTS AND DISCUSSION}

$\mathrm{SiO} 2$ has a thermal expansion coefficient of 0.5 $\times 10^{-6} / \mathrm{K}$, which is much smaller than the thermal expansion coefficient of Pt films $\left(8.8 \times 10^{-6} / \mathrm{K}\right)$. This would induce significant compressive stress in Pt (Ref. 14) when the Pt thin films were annealed. If the initial thickness of the metal is in the scale of nanometers, the compressive stress would cause the metal to form isolated islands. ${ }^{15}$ In our works, the thickness of the Pt films and the annealing temperature have been varied to produce Pt nanoparticles and Pt nanoisland films with different sized particle. Figure 2 shows the SEM photos
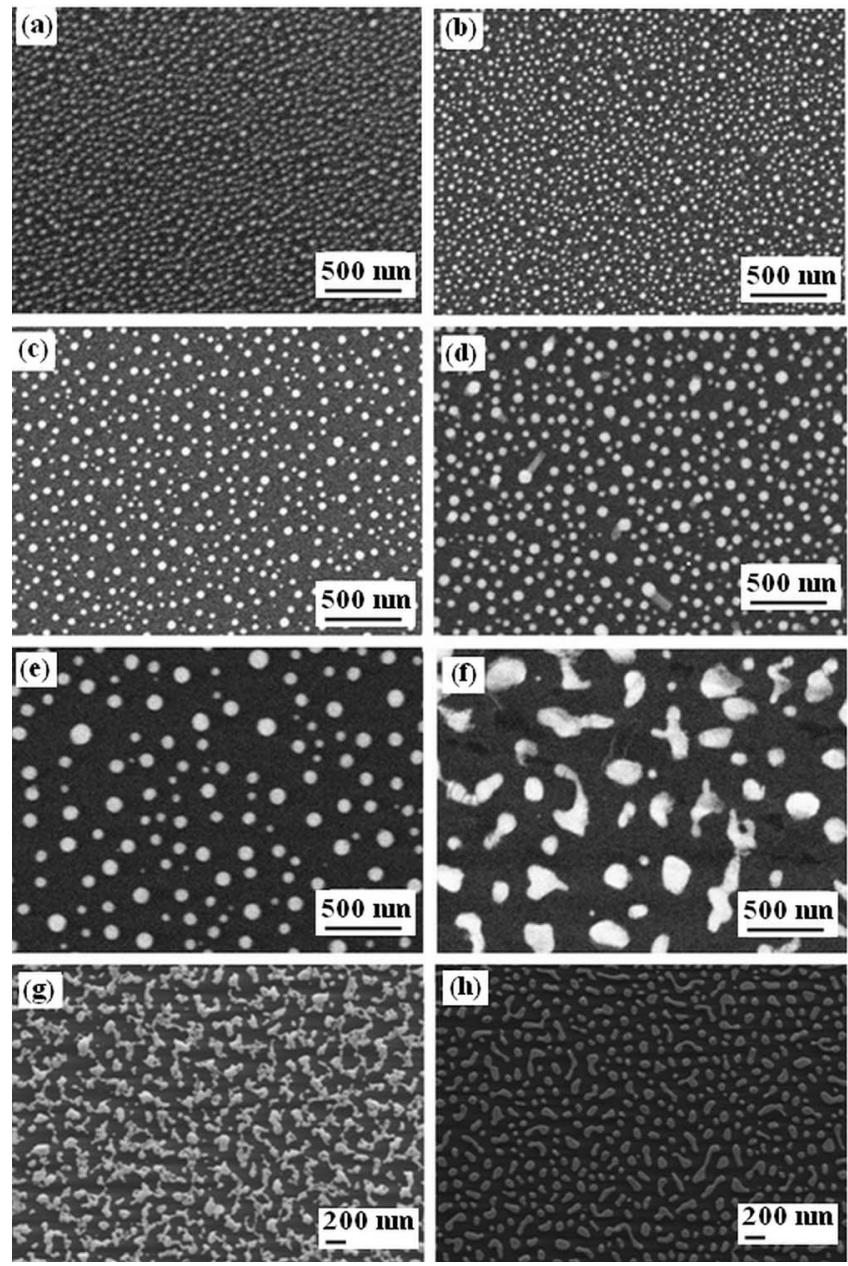

FIG. 2. SEM pictures of Pt nanoparticles and nanoisland films by different annealing methods. [(a)-(c)] $5 \mathrm{~nm}$ Pt thin film on the Si substrate annealed by the RTA process at 100, 1000, and $1200 \mathrm{~K}$, respectively. [(d) and (e)] $10 \mathrm{~nm}$ Pt thin film on Si substrate annealed by the RTA process at 1000 and $1200 \mathrm{~K}$, respectively. (f) $10 \mathrm{~nm}$ Pt thin film on Si substrate annealed by the vacuum process at $1000 \mathrm{~K}$. (g) $10 \mathrm{~nm}$ Pt thin film on quartz substrate annealed by the vacuum process at $1000 \mathrm{~K}$. (h) $10 \mathrm{~nm}$ Pt thin film on quartz substrate annealed by the RTA process at $1000 \mathrm{~K}$

of Pt nanoparticles and Pt nanoisland films with different sized particle. Figures 2(a)-2(c) are the SEM pictures of the $5 \mathrm{~nm} \mathrm{Pt}$ thin film on the Si substrate annealed by the RTA process at 1000, 1100, and $1200 \mathrm{~K}$, respectively. We found that the particle sizes increase with the annealing temperature. Figures 2(d) and 2(e) are the SEM pictures of the $10 \mathrm{~nm}$ $\mathrm{Pt}$ thin film on the Si substrate annealed by the RTA process at 1000 and $1200 \mathrm{~K}$, respectively. They also reveal that the particle sizes formed at the higher temperature are larger than those formed at the lower temperature. Annealing at the high temperature allows mass transport of small clusters across the surface to form larger clusters in a process analogous to the Ostwald ripening mechanism. ${ }^{16,17}$ Island formation mechanism analogous to Ostwald ripening could also be observed in our experiments. Figures 3(a) and 3(b) show feature variation in the surface morphology with the annealing temperature for those samples shown in Figs. 2(a)-2(e). Figure 3(a) demonstrates that average particle sizes increase with annealing temperature regardless whether the initial thickness of Pt is 5 or $10 \mathrm{~nm}$. Figure 3(b) shows that island 

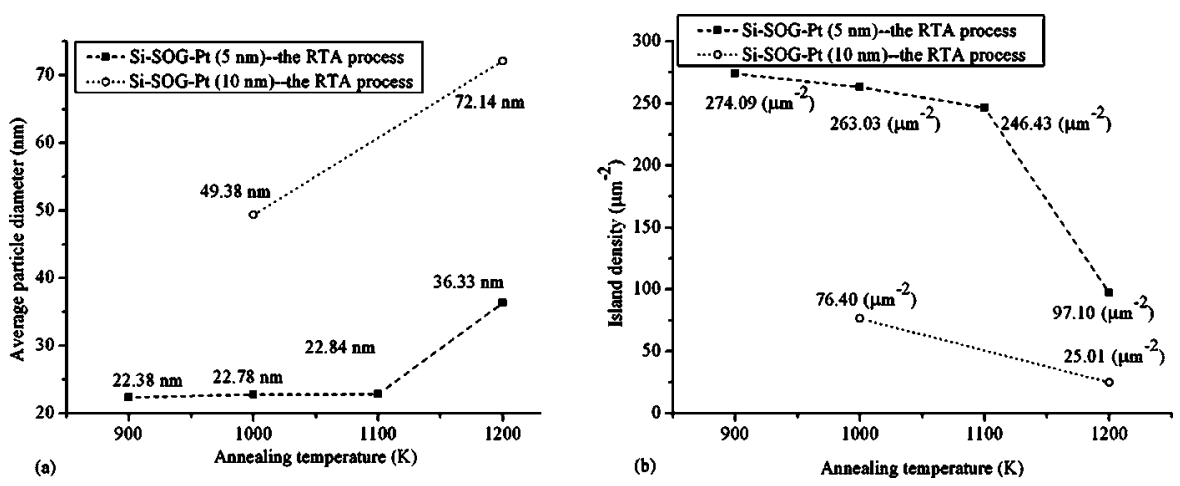

FIG. 3. (a) Average particle sizes at different annealing temperatures; (b) island density at different annealing temperatures.

density decreases with annealing temperature as well. It is clear that high annealing temperatures increase average particle size and reduce island density. Furthermore, Figs. 3(a) and 3(b) show that particle size suddenly increases and island density suddenly decreases at annealing temperatures above $1100 \mathrm{~K}$ for the $\mathrm{Pt}$ nanoparticles prepared with the $5 \mathrm{~nm}$ initial thickness. This may be attributed to surface melting of nanoparticles. ${ }^{18}$ The fact that nanoparticle size remains at around $23 \mathrm{~nm}$ and then abruptly increases to $36 \mathrm{~nm}$ after $1100 \mathrm{~K}$ indicates that the melting point of the $\mathrm{Pt}$ nanoparticles with average diameter of $23 \mathrm{~nm}$ might be around $1100 \mathrm{~K}$.

It is known that the initial thickness of the metal films can play an important role in determining their surface morphology at a given temperature. ${ }^{19}$ From Fig. 3(a), the average diameter of the $5 \mathrm{~nm}$ initial thickness of $\mathrm{Pt}$ annealed at $1000 \mathrm{~K}$ is $22.78 \mathrm{~nm}$. This is smaller than the $49.38 \mathrm{~nm} \mathrm{Pt}$ nanoparticles prepared with $10 \mathrm{~nm}$ initial thickness. Figure 3(b) shows that when Pt thin film is annealed at $1000 \mathrm{~K}$, the island densities are $263.03 \mu \mathrm{m}^{-2}$ for the sample with $5 \mathrm{~nm}$ initial thickness and $76.40 \mu \mathrm{m}^{-2}$ for the sample with $10 \mathrm{~nm}$ initial thickness. Similar results are shown in the Figs. 3(a) and 3(b) in which the samples were annealed at $1200 \mathrm{~K}$ as well. The thinner the initial film is, the larger the particle sizes and the higher the island density that are formed.

Previous studies have shown that the sizes of the metal islands are affected by different types of substrates ${ }^{6,20}$ and different ambiences during the annealing process. ${ }^{21}$ We investigated two processes, the vacuum process and the RTA process, and two different types of substrates, Si and quartz. Figures 2(f) and 2(g), respectively, show the SEM photos of the $10 \mathrm{~nm} \mathrm{Pt}$ thin film on the $\mathrm{Si}$ substrate and the quartz substrate annealed by the vacuum process at $1000 \mathrm{~K}$. Figures 2(d) and 2(h), respectively, show the SEM photos of the $10 \mathrm{~nm}$ Pt thin film on the Si substrate and the quartz substrate annealed by the RTA process at $1000 \mathrm{~K}$. Our experi- ments show that the island density of the Pt nanoisland films on quartz substrates is smaller than that on Si substrates both using the RTA and the vacuum process. In addition, the vacuum process results in a smaller island density than the RTA process, as shown in Table I.

The micro-PL of the Pt nanoparticles and the Pt nanoisland films was measured under the excitation of a $514.5 \mathrm{~nm}$ $\mathrm{Ar}^{+}$laser. Figure 4 shows the micro-PL spectra of several samples. Curves (a)-(c) are the micro-PL spectra of the Pt nanoparticles of different sizes prepared under different conditions. Curve (d) is the micro-PL of the sample of only SOG on $\mathrm{Si}$ without Pt. Curve (e) is the micro-PL spectrum of the $10 \mathrm{~nm} \mathrm{Pt}$ thin film on Si substrate but without annealing. Curves (d) and (e) are shown together for comparison. The luminescence from the Pt nanoparticles is largely enhanced, when compared to the spectrum of the $10 \mathrm{~nm} \mathrm{Pt}$ thin film without annealing. For instance, the integral intensity of the $49.38 \mathrm{~nm} \mathrm{Pt}$ nanoparticles with the micro-PL spectrum shown by curve (a) in Fig. 4 is about 38 times of that of the sample with the $10 \mathrm{~nm} \mathrm{Pt}$ thin film but without annealing. We also found that the peak wavelength of the micro-PL spectra of the Pt nanoparticles varies with their surface morphology. Similar phenomena have also been observed from gold nanoparticles and nanorods in previous studies. ${ }^{22-24}$ It has been demonstrated that the PL enhancement of noble metal nanoparticles or rough surfaces is due to local field enhancement. $^{7,22}$ Local field enhancement includes two parts: One is the collective oscillation of electrons in surface protrusions which can be induced by the optical field. This is referred to as the local plasmon effect. The other is that field tends to concentrate at the tips of surface protrusions. This is known as the lightning rod effect, which is the reason for the redshift of emission peaks with the increasing aspect ratio of nanorods. ${ }^{7,22,23}$ From our results, as shown in Fig. 4, we find that the peak wavelengths of curves (a) and (b) are close to $554 \mathrm{~nm}$, but the peak wavelength of curve (c) is significantly

TABLE I. Island formation conditions using the RTA and the vacuum processes.

\begin{tabular}{|c|c|c|c|c|c|}
\hline Substrates & $\begin{array}{l}\text { Initial } \\
\text { thickness } \\
\quad(\mathrm{nm})\end{array}$ & $\begin{array}{c}\text { Annealing } \\
\text { temperature } \\
(\mathrm{K})\end{array}$ & Process & $\begin{array}{l}\text { Island } \\
\text { density } \\
\left(\mu \mathrm{m}^{-2}\right)\end{array}$ & $\begin{array}{c}\text { SEM } \\
\text { image }\end{array}$ \\
\hline $\mathrm{Si}$ & 10 & 1000 & RTA & 76.40 & Fig. 2(d) \\
\hline $\mathrm{Si}$ & 10 & 1000 & Vacuum & 9.51 & Fig. 2(f) \\
\hline Quartz & 10 & 1000 & Vacuum & 9.12 & Fig. 2(g) \\
\hline Quartz & 10 & 1000 & RTA & 26.84 & Fig. 2(h) \\
\hline
\end{tabular}




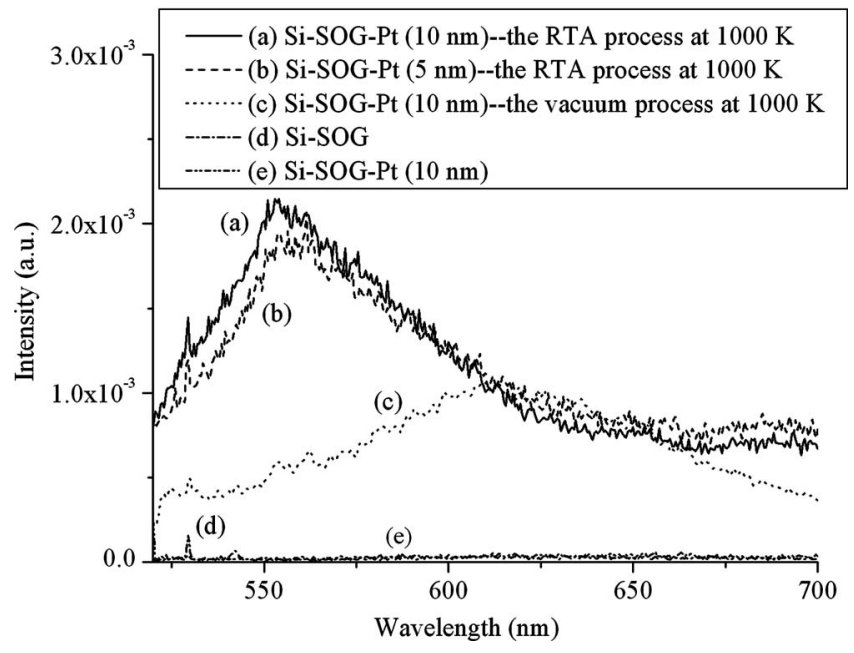

FIG. 4. The micro-PL spectra of samples on Si substrates with (a) the $10 \mathrm{~nm}$ Pt thin film annealed by the RTA process at $1000 \mathrm{~K}$, (b) the $5 \mathrm{~nm} \mathrm{Pt}$ thin film annealed by the RTA process at $1000 \mathrm{~K}$, (c) the $10 \mathrm{~nm}$ Pt thin film annealed by the vacuum process at $1000 \mathrm{~K}$, (d) the SOG film, and (e) the $10 \mathrm{~nm}$ Pt thin film without annealing.

redshifted to $610 \mathrm{~nm}$. The particles whose micro-PL spectra are shown in Figs. 4(a) and 4(b) are almost round in shape. The aspect ratio of the Pt nanoparticles is obviously smaller than that of the Pt nanoisland films. As a result, the redshift of the peak wavelength of the micro-PL from the Pt nanoisland films is to be expected. We suggest that the variation of the peak wavelength of the micro-PL from $\mathrm{Pt}$ is due to the lightning rod effect.

The enhanced micro-PL of $\mathrm{Pt}$ can be found in the $\mathrm{Pt}$ nanoisland films on the quartz substrates as well, as shown in Fig. 5 In Figs. 5(a) and 5(b) are shown the micro-PL spectra of the Pt nanoisland film formed by the RTA process and the vacuum process, respectively. Curve (c) is the micro-PL spectra of the $10 \mathrm{~nm} \mathrm{Pt}$ thin film on the quartz substrate without annealing. From Fig. 5, the integral intensity of the micro-PL spectrum shown by curve (a) for the Pt nanoisland film (island density of $26.84 \mu \mathrm{m}^{-2}$ ) is about eight times of

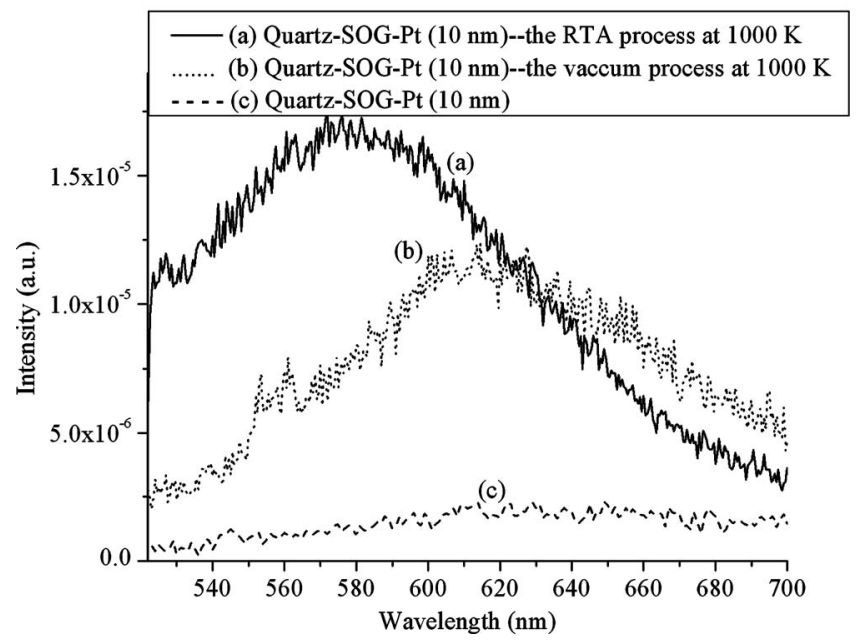

FIG. 5. The micro-PL spectra of the samples on quartz substrates with (a) the $10 \mathrm{~nm}$ Pt thin film annealed by the RTA process at $1000 \mathrm{~K}$, (b) the $10 \mathrm{~nm}$ Pt thin film annealed by the vacuum process at $1000 \mathrm{~K}$, and (c) the $10 \mathrm{~nm}$ Pt thin film without annealing.

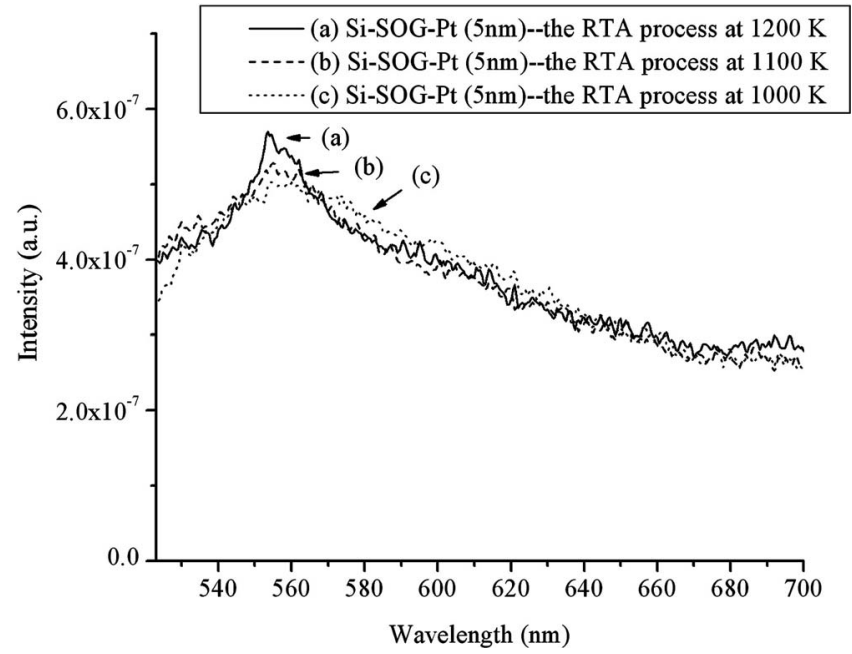

FIG. 6. The micro-PL spectra for the samples with $5 \mathrm{~nm}$ initial thickness annealed at different temperatures by the RTA process. (a) The annealing temperature is $1200 \mathrm{~K}$. (b) The annealing temperature is $1100 \mathrm{~K}$. (c) The annealing temperature is $1000 \mathrm{~K}$.

that of the sample with the $10 \mathrm{~nm}$ Pt thin film without annealing. In addition, the variation of the peak wavelength of the micro-PL due to different surface morphologies of Pt can be found. The peak wavelength of the micro-PL of the Pt nanoisland film annealed by the RTA process is about $576 \mathrm{~nm}$, and for the sample annealed by the vacuum process, it is close to $615 \mathrm{~nm}$. Figure 6 shows the micro-PL spectra of the samples with $5 \mathrm{~nm}$ initial thickness of Pt annealed at different temperatures. Curves (a)-(c) in Fig. 6 show the samples annealed by the RTA process at 1200, 1100, and $1000 \mathrm{~K}$, respectively. The peak wavelengths of the micro-PL spectra are all close to $554 \mathrm{~nm}$. This is due to the fact that the particles of the samples with the $5 \mathrm{~nm}$ initial thickness of $\mathrm{Pt}$ annealed at temperatures higher than $1000 \mathrm{~K}$ are almost round. This micro-PL is significantly enhanced by the Pt nanomorphology.

Similar results showing intense fluorescence from transition-metal nanoparticles such as $\mathrm{Fe}$ have been reported. ${ }^{25}$ The authors suggest that the partial oxidation of metal nanoparticles possibly occur on the metal's surface. These metal oxide clusters are photoactivated and might give rise to the observed fluorescence. In order to confirm the composition of the Pt nanoparticles and the nanoisland films, the XPS and XRD measurements are utilized. The XPS studies were carried out with a $\mathrm{Mg} K \alpha$ X-ray source $(1253.6 \mathrm{eV})$. One of our samples' XPS spectra is shown in Fig. 7. This illustrates the sample with $10 \mathrm{~nm}$ Pt thin film annealed by the RTA process at $1000 \mathrm{~K}$. The XPS peaks in Fig. 7 are referenced to the $\mathrm{C} 1 \mathrm{~s}$ binding energy of the adventitious carbon at $284.5 \mathrm{eV}$. Figure 7(a) is the XPS spectrum in the range between 0 and $600 \mathrm{eV}$. All the peaks are labeled and reveal that three elements, $\mathrm{Pt}, \mathrm{Si}$, and $\mathrm{O}$, exist at the surface of the samples. To get a clear view, the XPS spectrums of Si $2 p$ and Pt $4 f$ are plotted in Figs. 7(b) and 7(c), respectively. From Fig. 7(b), the peak of $\mathrm{Si} 2 p$ is at $103.43 \mathrm{eV}$, which is originated from the SOG film under the Pt nanoparticles. From Fig. 7(c), the peak of Pt $4 f_{7 / 2}$ is at $71.11 \mathrm{eV}$. The fact that the binding energy of the $\mathrm{Pt} 4 f_{7 / 2}$ peak for the bulk Pt measured 

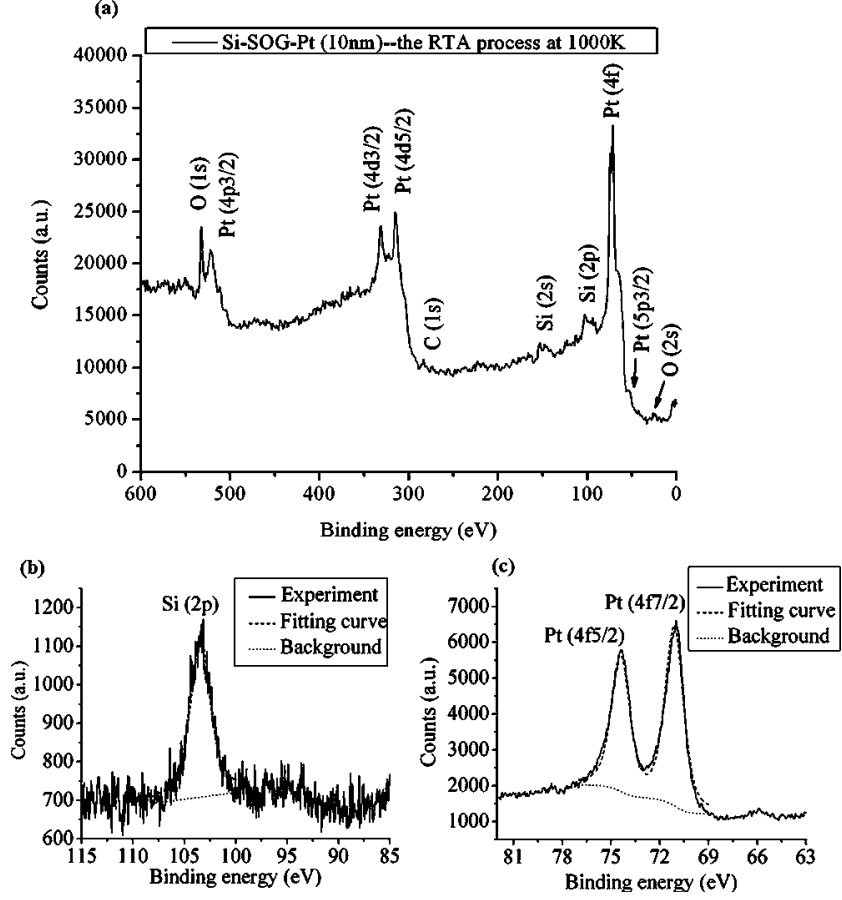

FIG. 7. The XPS spectra for the sample with the $10 \mathrm{~nm}$ Pt thin film annealed by the RTA process at $1000 \mathrm{~K}$. (a) The XPS spectrum in the range of 0-600 eV. (b) The XPS spectrum for Si $2 p$. (c) The XPS spectrum for Pt $4 f$.

by the same instrument is $71.3 \mathrm{eV}$ suggests that oxidation and silicide formation on Pt nanoparticles during annealing do not occur. Figure 8 shows the result of the XRD of the Pt nanoisland films on the quartz substrates annealed by different processes. Only the XRD signal corresponding to $\mathrm{Pt}$ (111) is found. The broad XRD signal of $a-\mathrm{PtO}_{x}\left(2 \theta=32^{\circ}\right)$ (Ref. 26) and the XRD signals of PtSi or $\mathrm{Pt}_{2} \mathrm{Si}$ (Ref. 27) are not observed. The results show that the SOG films under the Pt thin film could prevent the formation of platinum silicide and platinum oxide during annealing. This result is in line with previous research. ${ }^{13}$ The investigation using the XPS and XRD measurements suggests that the composition of the

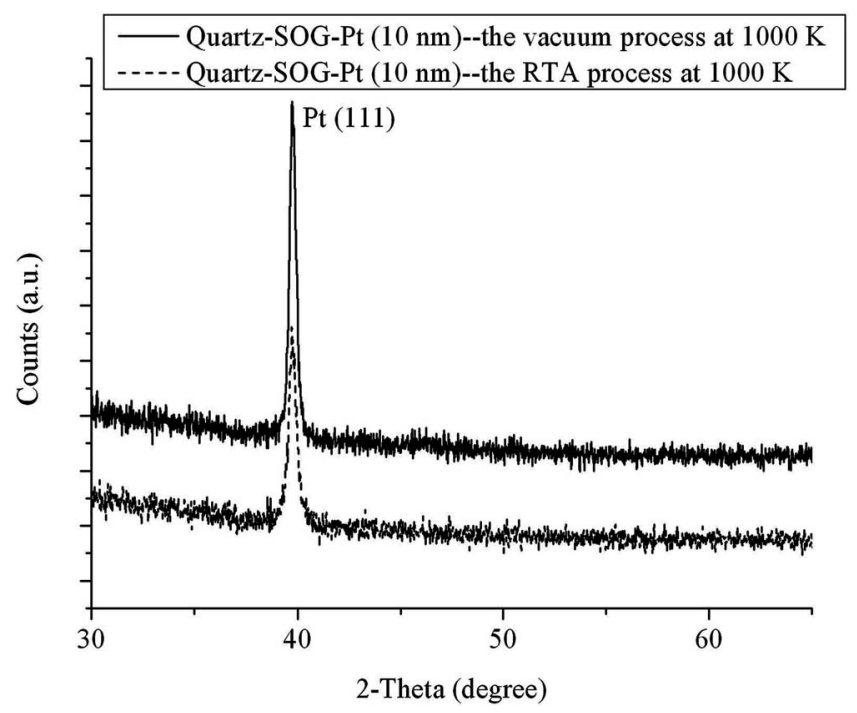

FIG. 8. The XRD spectra of the Pt nanoisland films annealed by different processes. Only the peak corresponding to $\mathrm{Pt}(111)$ is found. nanoisland films and the nanoparticles mainly consist of Pt. As a result, we believe that their optical properties as measured by micro-PL should be related to their surface morphology.

Since the results of the XPS and the XRD show that the nanoparticles and the nanoisland films are composed of $\mathrm{Pt}$, we suggest that the origin of micro-PL from Pt might be attributed to $d$-band and $s p$-band transitions. The mechanism is analogous to that of noble metals. The characteristics of the $d$ band of $\mathrm{Pt}$ are not like those of noble metals, which lie deeply under the Fermi level. The $d$ band of Pt extends into the Fermi level and over about $0.3 \mathrm{eV}^{28,29}$ Since the Coulomb carrier-carrier scattering time considerably depends on the density of states (DOS) around the Fermi level, and the DOS around the Fermi level is enhanced by the $d$ states in Pt, the lifetime of the photoexcited carriers is smaller in Pt than in noble metals. ${ }^{30}$ This significantly reduces the radiative electron-hole recombination in the smooth Pt film. For this reason, the signal of emission is very low and could not be easily detected from the Pt thin films in our experiment. However, as nanoparticles and the nanoisland films are formed after annealing, the efficiency of the light coming from Pt can be greatly improved because of the local field enhancement mechanism.

The extinction spectra of Pt nanoisland films on quartz substrates were measured to observe the localized surface plasmon effect in our samples with the quartz substrates. Localized surface plasmon resonance (LSPR) is the excitation of collective resonance of conduction electrons induced by electromagnetic radiation. ${ }^{31-33}$ The frequency of the LSPR can be tuned by changing the size, shape, and dielectric constant of the surrounding medium. ${ }^{31-33}$ For platinum, previous studies had shown that the frequency of the LSPR is located in the ultraviolet light region. ${ }^{13,22}$ Nonetheless, recently, Langhammer et al. ${ }^{33}$ reported that the extinction peak of a Pt nanodisk would shift from 200 to $1800 \mathrm{~nm}$ as the diameters varied from 38 to $530 \mathrm{~nm}$. Figures 9(a) and 9(b) are the extinction spectra of the samples with the $10 \mathrm{~nm} \mathrm{Pt}$ thin film annealed by the RTA and the vacuum processes at $1000 \mathrm{~K}$. The SOG film on the quartz substrate is used as a reference, indicating that the extinction features result from the platinum nanoisland films. Figure 9(a) shows that there are extinction peaks at 230 and $330 \mathrm{~nm}$ for the sample annealed by the RTA process at $1000 \mathrm{~K}$. Figure 9(b) shows that the extinction peaks are at 330 and $512 \mathrm{~nm}$ for the sample annealed by the vacuum process at $1000 \mathrm{~K}$. These peaks are referred to as the frequency of the LSPR of the Pt nanoisland films. The characteristics of the two peaks in the extinction spectra might be attributed to the resonant frequency of the transverse and the longitudinal plasmon oscillation. ${ }^{22-24}$ Since the frequency of the LSPR of metal nanostructures is redshifted with increasing particle size and aspect ratio, ${ }^{22-24,33}$ the extinction spectra in Figs. 9(a) and 9(b) reveal that the particle size and the aspect ratio of the $\mathrm{Pt}$ nanoisland films annealed by the vacuum process are larger than those of the Pt nanoisland films annealed by the RTA process. This result corresponds to their surface morphology, as shown in the SEM pictures [Figs. 2(g) and 2(h)] and their micro-PL behaviors [curves (a) and (b) in Fig. 5]. In addi- 

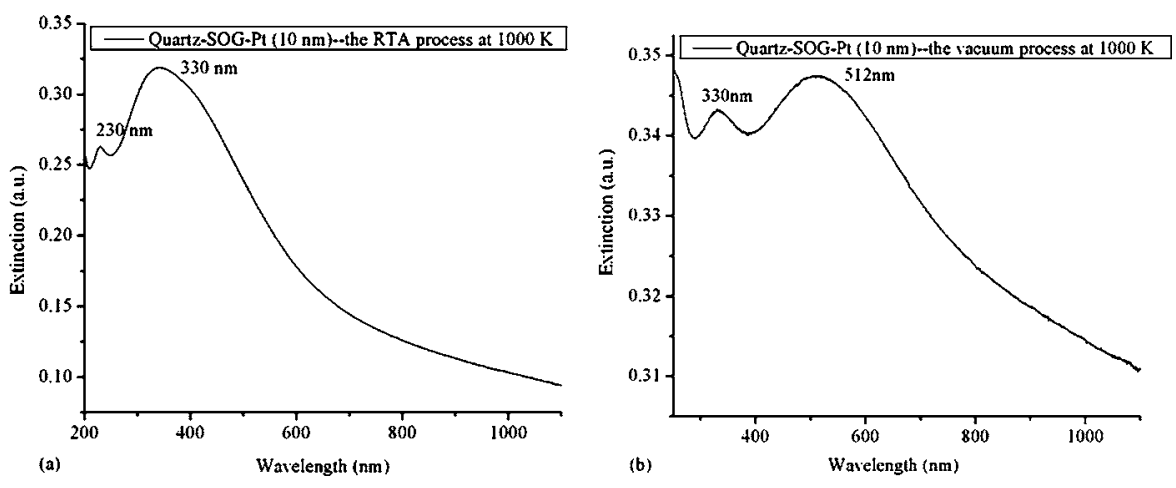

FIG. 9. The extinction spectra of the samples on quartz substrates with (a) the $10 \mathrm{~nm}$ Pt thin film annealed by the RTA process at $1000 \mathrm{~K}$ and (b) the $10 \mathrm{~nm}$ Pt thin film annealed by the vacuum process at $1000 \mathrm{~K}$. tion, we could not find a peak near $1.2-1.4 \mathrm{eV}$ from the extinction spectra, which is assigned to the absorption peak of $\mathrm{PtO}_{x}{ }^{34,35}$ We can confirm that the extinction features result from the surface morphology of Pt.

Some research has suggested that the PL of noble metals might be related to the resonance of surface plasmon. Dulkeith et $a l .^{8}$ reported that the emission spectra of gold nanoparticles could coincide with their extinction spectra according to the Mie theory, so they stated that PL enhancement should be due to the emission of particle plasmons. Mohamed et al. ${ }^{23}$ suggested that the relaxation of surface plasmon coherent electronic motions followed by the recombination of the $d$-band holes and the $s p$-band electrons lead to the enhanced PL of gold since the wavelength of the LSPR was close to that of their excitation laser. However, Varnavski et al. ${ }^{24}$ found that the luminescence efficiency is the same for gold nanospheres (with a surface plasmon resonance of $520 \mathrm{~nm}$ ) when using an excitation laser at 4.65 and $3.02 \mathrm{eV}$. Their experimental results are inconsistent with the suggestion that the observed emission is due to surface plasmon emission or surface plasmon excitation by incoming light. They reported that the emission originated from the $s p$-electron/ $d$-hole recombination with the enhancement of the incoming and outgoing fields via the local field enhancement mechanism. From our extinction spectra, as shown in Fig. 9, one of the LSPR for the Pt nanoisland films annealed by the vacuum process is $512 \mathrm{~nm}$. This is close to the wavelength of our excitation laser $(514.5 \mathrm{~nm})$. This result shows the possibility that the micro-PL enhancement could be related to the excitation of the LSPR. However, for the sample annealed by the RTA process, the LSPR differs greatly from the wavelength of our excitation laser and the peak position of the micro-PL. If the observed micro-PL comes from LSPR excitation, the signal of micro-PL from the sample annealed by the vacuum process should be larger than that from the sample annealed by the RTA process. Nevertheless, our experimental results contradict this. The integral intensity of the micro-PL for the sample annealed by the vacuum process is lower than that of the sample annealed by the RTA process. These results reveal that the observed micro-PL of $\mathrm{Pt}$ would not be significantly influenced by the behaviors of the LSPR. The excitation of the LSPR might contribute to the enhancement of micro-PL for the sample annealed by the vacuum process, but whether the LSPR is crucial to the observed micro-PL could not be easily confirmed by our inves- tigation. As a result, we suggest that the observed micro-PL of Pt is attributed to the local field enhancement of the incoming and outgoing fields.

\section{CONCLUSIONS}

In summary, we used the annealing process to produce Pt nanoparticles or Pt nanoisland films of different sizes as a function of temperature, film thickness, pressure, and underlying substrates. The high annealing temperature caused particle size to increase and island density to decrease. Increasing the initial thickness of Pt from 5 to $10 \mathrm{~nm}$, we found that the particle sizes doubled and island density was reduced. The quartz substrates resulted in a smaller island density than the $\mathrm{Si}$ substrates and the vacuum process produced a smaller island density than the RTA process. Furthermore, our experiment suggests that the melting point of the $23 \mathrm{~nm} \mathrm{Pt}$ nanoparticles is about $1100 \mathrm{~K}$. In addition, we found that the surface enhanced micro-PL of Pt is analogous to the PL of noble metal nanoparticles. The integral intensity of the micro-PL of the $49.38 \mathrm{~nm}$ Pt nanoparticles is 38 times of that of the Pt thin film. The peak wavelength of the micro-PL of the Pt nanoparticles varied from 554 to $615 \mathrm{~nm}$ as a result of the change in the Pt surface morphology. Extinction spectra show that the frequency of the LSPR for the Pt nanoisland film annealed by the RTA process is at 230 and $330 \mathrm{~nm}$. Those peaks redshift to 330 and $512 \mathrm{~nm}$ for the Pt nanoisland film annealed by the vacuum process. The redshift of the extinction peaks reveals that the particle size and the aspect ratio of the $\mathrm{Pt}$ nanoisland film annealed by the vacuum process are larger than those of the Pt nanoisalnd film annealed by the RTA process. This result corresponds to their surface morphology, as shown by the SEM pictures and their micro-PL behaviors. From our studies of the XPS and the XRD, we suggest that the observed optical properties of the Pt nanoparticles and the nanoisland films are related to their surface morphology. Our results for the optical properties of Pt nanoparticles measured by micro-PL and extinction could lead to a wide range of potential applications, including solar cells and surface enhanced Raman scattering.

\section{ACKNOWLEDGMENT}

This work is supported by the National Science Council, Taiwan, Republic of China, with Grant Nos. NSC95-2120M-002-001 and NSC95-2112-M-002-004. 
${ }^{1}$ M. M. Alvarez, J. T. Khoury, T. G. Schaaff, M. N. Shafigullin, I. Vezmar, and R. L. Whetten, J. Phys. Chem. B 101, 3706 (1997).

${ }^{2}$ A. M. Michaels, M. Nirmal, and L. E. Brus, J. Am. Chem. Soc. 121, 9932 (1999).

${ }^{3}$ M. Moscovits, Rev. Mod. Phys. 57, 783 (1985).

${ }^{4}$ A. Mooradian, Phys. Rev. Lett. 22, 185 (1969).

${ }^{5}$ T. Qiu, X. L. Wu, and Y. C. Cheng, Appl. Phys. Lett. 88, 143111 (2006).

${ }^{6}$ L. Khriachtchev, L. Heikkila, and T. Kuusela, Appl. Phys. Lett. 78, 1994 (2001).

${ }^{7}$ G. T. Boyd, Z. H. Yu, and Y. R. Shen, Phys. Rev. B 33, 7923 (1986).

${ }^{8}$ E. Dulkeith, T. Niedereichholz, T. A. Klar, J. Feldmann, G. von Plessen,

D. I. Gittins, K. S. Mayya, and F. Caruso, Phys. Rev. B 70, 205424 (2004)

${ }^{9}$ Z. Q. Tian, B. Ren, and D. Y. Wu, J. Phys. Chem. B 106, 9463 (2002).

${ }^{10}$ W. B. Cai, B. Ren, X. Q. Li, C. X. She, F. M. Liu, X. W. Cai, and Z. Q. Tian, Surf. Sci. 406, 9 (1998).

${ }^{11}$ A. E. Bjerke and P. R. Griffiths, Appl. Spectrosc. 56, 1275 (2002).

${ }^{12}$ J. M. Lin, H. Y. Lin, C. L. Cheng, and Y. F. Chen, Nanotechnology 17, 4391 (2006).

${ }^{13}$ E. Anno and M. Tanimoto, J. Appl. Phys. 88, 3426 (2000).

${ }^{14}$ C. Bower, O. Zhou, W. Zhu, D. J. Werder, and S. Jin, Appl. Phys. Lett. 77, 2767 (2000).

${ }^{15}$ J. A. Floro, S. J. Hearne, J. A. Hunter, P. Kotula, E. Chason, S. C. Seel, and C. V. Thompson, J. Appl. Phys. 89, 4886 (2001).

${ }^{16}$ J. D. Carey, L. L. Ong, and S. R. P. Silva, Nanotechnology 14, 1223 (2003).

${ }^{17}$ S. Wei, B. Li, T. Fujimoto, and I. Kojima, Phys. Rev. B 58, 3605 (1998).

${ }^{18}$ Z. L. Wang, J. M. Petroski, T. C. Green, and M. A. El-Sayed, J. Phys. Chem. B 102, 6145 (1998).
${ }^{19}$ S. Aggarwal, S. B. Ogale, C. S. Ganpule, S. R. Shinde, V. A. Novikov, A. P. Monga, M. R. Burr, R. Ramesh, V. Ballarotto, and E. D. Williams, Appl. Phys. Lett. 78, 1442 (2001).

${ }^{20}$ T. L. Alford, L. Chen, and K. S. Gadre, Thin Solid Films 429, 248 (2003).

${ }^{21}$ A. S. Eppler, G. Rupprechter, E. A. Anderson, and G. A. Somorjai, J. Phys. Chem. B 104, 7286 (2000).

${ }^{22}$ S. Link and M. A. El-Sayed, Annu. Rev. Phys. Chem. 54, 331 (2003).

${ }^{23}$ M. B. Mohamed, V. Volkov, S. Link, and M. A. El-Sayed, Chem. Phys. Lett. 317, 517 (2000).

${ }^{24}$ O. P. Varnavski, T. Goodson III, M. B. Mohamed, and M. A. El-Sayed, Phys. Rev. B 72, 235405 (2005).

${ }^{25}$ A. Alqudami and S. Annapoorni, Plasmonics 2, 5 (2007).

${ }^{26}$ K. L. Saenger, C. Cabral, Jr., C. Lavoie, and S. M. Rossnagel, J. Appl. Phys. 86, 6084 (1999).

${ }^{27}$ A. A. Naem, J. Appl. Phys. 64, 4161 (1988).

${ }^{28}$ N. V. Smith, Phys. Rev. B 9, 1365 (1974).

${ }^{29}$ R. E. Dietz, E. G. McRae, and J. H. Weaver, Phys. Rev. B 21, 2229 (1980).

${ }^{30}$ N. Pontius, M. Neeb, W. Eberhardt, G. Luttgens, and P. S. Bechthold, Phys. Rev. B 67, 035425 (2003).

${ }^{31}$ D. Dalacu and L. Martinu, J. Opt. Soc. Am. B 18, 85 (2001)

${ }^{32}$ A. Hilgera, N. Cuppers, M. Tenfelde, and U. Kreibig, Eur. Phys. J. D 10, 115 (2000).

${ }^{33}$ C. Langhammer, Z. Yuan, I. Zoric, and B. Kasemo, Nano Lett. 6, 833 (2006).

${ }^{34}$ H. Neff, S. Henkel, E. Hartmannsgruber, E. Steinbeiss, W. Michalke, K. Steenbeck, and H. G. Schmidt, J. Appl. Phys. 79, 7672 (1996).

${ }^{35}$ C. R. Aita and Ngoc C. Tran, J. Appl. Phys. 56, 958 (1984). 